\title{
The Ostrogradsky series and related Cantor-like sets
}

\author{
by
}

Sergio Albeverio (Bonn), Oleksandr Baranovskyi (Kyiv), Mykola Pratsiovytyi (Kyiv) and Grygoriy Torbin (Bonn and Kyiv)

Introduction. There are many different methods of expansion and encoding (representation) of real numbers by using a finite or an infinite alphabet $A$. The $s$-adic expansions, continued fractions, $f$-expansions, the Lüroth expansions etc. are widely used in mathematics (see, e.g., [17]). Each representation has its own features and generates its own "geometry" and metric theory. To each representation there is associated a system of cylindrical sets, partitioning the unit interval (or the real line). From the ratios of the lengths of cylindrical sets the basic metric relations follow (in the form of equalities and inequalities) which are crucial for the development of the corresponding metric theory, i.e., a theory about measure (e.g., Jordan, Lebesgue, Hausdorff, Hausdorff-Billingsley, ... ) of sets of real numbers defined by characteristic properties of their digits in the corresponding representation (see, e.g., $[1,2,6,9,10,15,17])$.

The present paper is devoted to the investigation of the expansion of real numbers in the first Ostrogradsky series (introduced by M. V. Ostrogradsky (1801-1862), a well known Ukrainian mathematician). In this case the alphabet $A$ coincides with the set $\mathbb{N}$ of positive integers.

The expansion

$$
x=\frac{1}{q_{1}}-\frac{1}{q_{1} q_{2}}+\cdots+\frac{(-1)^{n-1}}{q_{1} q_{2} \ldots q_{n}}+\cdots,
$$

where the $q_{n}$ are positive integers and $q_{n+1}>q_{n}$ for all $n$, is said to be the expansion of $x$ in the first Ostrogradsky series. The expansion

$$
x=\frac{1}{q_{1}}-\frac{1}{q_{2}}+\cdots+\frac{(-1)^{n-1}}{q_{n}}+\cdots,
$$

where the $q_{n}$ are positive integers and $q_{n+1} \geq q_{n}\left(q_{n}+1\right)$ for all $n$, is said

2000 Mathematics Subject Classification: 11K55, 11K50.

Key words and phrases: Ostrogradsky representation of real numbers, Cantor-like sets, Lebesgue measure, continued fractions. 
to be the expansion of $x$ in the second Ostrogradsky series. Each irrational number has a unique expansion of the form (1) or (2). Rational numbers have two different finite representations of the above forms (see, e.g., [16]).

Equality (1) can be rewritten as

$$
x=\frac{1}{g_{1}}-\frac{1}{g_{1}\left(g_{1}+g_{2}\right)}+\cdots+\frac{(-1)^{n-1}}{g_{1}\left(g_{1}+g_{2}\right) \cdots\left(g_{1}+\cdots+g_{n}\right)}+\cdots,
$$

where $g_{1}=q_{1}, g_{n+1}=q_{n+1}-q_{n}$ for any $n \in \mathbb{N}$. The expression (3) is said to be the $\overline{\mathrm{O}}^{1}$-representation and the number $g_{n}=g_{n}(x)$ is the $n$th $\overline{\mathrm{O}}^{1}$-symbol of $x$.

Shortly before his death, M. V. Ostrogradsky found two algorithms for the representation of real numbers via alternating series of the form (1) and (2), but he did not publish them. Short unpublished remarks of Ostrogradsky concerning the above representations have been found by E. Ya. Remez [16]. Some similarities between the Ostrogradsky series and continued fractions have been pointed out in the same paper. E. Ya. Remez also dealt with applications of the Ostrogradsky series to numerical solution of algebraic equations. In the editorial comments to the book [6] B. V. Gnedenko has pointed out that there are no fundamental investigations of properties of the above mentioned representations. Analogous problems were studied by W. Sierpiński [18] and T. A. Pierce [11] independently. Some algorithms for representation of real numbers by means of positive and alternating series were proposed in [18]. Two of these algorithms lead to the Ostrogradsky series (1) and (2). An algorithm leading to the representation of irrational numbers in the form (1) has also been considered in [11]. An algorithm for a general alternating series expansion for real numbers in terms of rationals has been considered in [5], where the so-called alternating Lüroth and modified Engel-type expansions were also studied. This algorithm also leads to expansions of real numbers in Ostrogradsky series.

Let us mention some papers devoted to applications of the Ostrogradsky series. Connections between the Ostrogradsky algorithms and the algorithm for continued fractions have been established in [4]. This book also contains generalizations of the above algorithms. In [7] different types of $p$-adic continued fractions have been constructed on the basis of $p$-adic analogs of the Euclid and Ostrogradsky algorithms. Combining the algorithms of Engel and Ostrogradsky in a special way, the same author [8] has constructed an algorithm for representation of real numbers via series which converge faster than the corresponding Engel and Ostrogradsky series. The paper [19] is devoted to the investigation of the first Ostrogradsky algorithm and to the determination of the expectation of the random variables $\left(q_{j}+1\right)^{\nu}$, $\nu \geq 0$, and $r_{n}=\sum_{j=n+1}^{\infty}(-1)^{j+1} / q_{1} \ldots q_{j}$, where the $q_{j}=q_{j}(\alpha)$ are random variables depending on the random variable $\alpha$, uniformly distributed 
on the unit interval. In the same paper a generalization of the Ostrogradsky algorithm to approximations in Banach spaces has been proposed.

In this paper we continue to study the "geometry" of the representation generated by the first Ostrogradsky series $[3,13,14]$. In Section 1 we prove basic metric relations for the $\overline{\mathrm{O}}^{1}$-representation and compare them with the corresponding relations for continued fractions. Section 2 is devoted to the study of the set $C\left[\overline{\mathrm{O}}^{1},\left\{V_{n}\right\}\right]$, consisting of the real numbers whose $n$th $\overline{\mathrm{O}}^{1}$ symbols take values from the set $V_{n} \subset \mathbb{N}$, for each $n \in \mathbb{N}$. Conditions for the set $C\left[\overline{\mathrm{O}}^{1},\left\{V_{n}\right\}\right]$ to be of zero resp. positive Lebesgue measure $\lambda$ are found. In particular, we prove that $\lambda\left(C\left[\overline{\mathrm{O}}^{1},\left\{V_{n}\right\}\right]\right)>0$ if $V_{n}=V=\{m+1, m+2, \ldots\}$, where $m$ is an arbitrary positive integer. This marks an essential difference between the metric theories of continued fractions and $\overline{\mathrm{O}}^{1}$-representations.

\section{Representations of real numbers by the Ostrogradsky series}

Definition 1. A finite or an infinite expression

$$
\sum_{n} \frac{(-1)^{n-1}}{q_{1} \ldots q_{n}}=\frac{1}{q_{1}}-\frac{1}{q_{1} q_{2}}+\cdots,
$$

where the $q_{n}$ are positive integers and $q_{n+1}>q_{n}$ for all $n$, is called the first Ostrogradsky series (hereafter, the Ostrogradsky series). The numbers $q_{n}$ are called the elements of the Ostrogradsky series (4).

We denote the expression (4) briefly by $\mathrm{O}^{1}\left(q_{1}, \ldots, q_{n}\right)$ if it contains a finite number of terms, and we speak in this case of a finite Ostrogradsky series. We write $\mathrm{O}^{1}\left(q_{1}, q_{2}, \ldots\right)$ in the case of infinitely many terms.

It is known (see, e.g., [16]) that every Ostrogradsky series is convergent and its sum belongs to $[0,1]$, and any real number $x \in(0,1)$ can be represented in the form (4). If $x$ is irrational then the expression (4) is unique and it has an infinite number of terms. If $x$ is rational then it can be represented in the form (4) in two different ways:

$$
x=\mathrm{O}^{1}\left(q_{1}, \ldots, q_{n-1}, q_{n}, q_{n}+1\right)=\mathrm{O}^{1}\left(q_{1}, \ldots, q_{n-1}, q_{n}+1\right) .
$$

We can find the elements of the Ostrogradsky series for a given number $x$ using the following algorithm:

$$
\begin{aligned}
& 1=q_{1} x+\alpha_{1} \quad\left(0 \leq \alpha_{1}<x\right), \\
& 1=q_{2} \alpha_{1}+\alpha_{2} \quad\left(0 \leq \alpha_{2}<\alpha_{1}\right), \\
& 1=q_{n} \alpha_{n-1}+\alpha_{n} \quad\left(0 \leq \alpha_{n}<\alpha_{n-1}\right),
\end{aligned}
$$


Let

$$
g_{1}=q_{1}, \quad g_{n+1}=q_{n+1}-q_{n} \quad \text { for any } n \in \mathbb{N} .
$$

Then one can rewrite (4) in the form

$$
\sum_{n} \frac{(-1)^{n-1}}{g_{1}\left(g_{1}+g_{2}\right) \ldots\left(g_{1}+\cdots+g_{n}\right)}=\frac{1}{g_{1}}-\frac{1}{g_{1}\left(g_{1}+g_{2}\right)}+\cdots .
$$

We denote the expression (5) by $\overline{\mathrm{O}}^{1}\left(g_{1}, g_{2}, \ldots\right)$. A representation of a number $x \in(0,1)$ by $(5)$ is called the $\overline{\mathrm{O}}^{1}$-representation and $g_{n}=g_{n}(x)$ is the $n$th $\overline{\mathrm{O}}^{1}$-symbol of $x$.

Let $c_{1}, \ldots, c_{m}$ be a fixed sequence of positive integers.

Definition 2. The set $\overline{\mathrm{O}}_{\left[c_{1} \ldots c_{m}\right]}^{1}$, which is the closure of the set of all $x \in(0,1)$ whose first $m \overline{\mathrm{O}}^{1}$-symbols are $c_{1}, \ldots, c_{m}$ is said to be the cylindrical set (cylinder) of rank $m$ with base $\left(c_{1}, \ldots, c_{m}\right)$, i.e.,

$$
\overline{\mathrm{O}}_{\left[c_{1} \ldots c_{m}\right]}^{1}=\left(\left\{x: x=\overline{\mathrm{O}}^{1}\left(g_{1}(x), \ldots\right), g_{k}(x)=c_{k}, 1 \leq k \leq m\right\}\right)^{\mathrm{cl}} .
$$

It is not hard to prove that $\overline{\mathrm{O}}_{\left[c_{1} \ldots c_{m}\right]}^{1}$ is a closed interval of length

$$
\left|\overline{\mathrm{O}}_{\left[c_{1} \ldots c_{m}\right]}^{1}\right|=\frac{1}{\sigma_{1} \ldots \sigma_{m}\left(\sigma_{m}+1\right)},
$$

where $\sigma_{k}=\sum_{i=1}^{k} c_{i}$.

REMARK. We shall denote by $\overline{\mathrm{O}}_{\left(c_{1} \ldots c_{m}\right)}^{1}$ the interior of $\overline{\mathrm{O}}_{\left[c_{1} \ldots c_{m}\right]}^{1}$.

Let us mention that the cylindrical set $\overline{\mathrm{O}}_{[\underbrace{11 \ldots 1}_{m}]}^{1}$ has the largest length among all cylindrical sets of rank $m$, namely

$$
\left|\overline{\mathrm{O}}_{[\underbrace{11 \ldots 1}_{m}}^{1}\right|=\frac{1}{(m+1) !},
$$

and there exist cylindrical sets of different ranks with the same length. For instance,

$$
\left|\overline{\mathrm{O}}_{[1 c]}^{1}\right|=\left|\overline{\mathrm{O}}_{[c+1]}^{1}\right|, \quad\left|\overline{\mathrm{O}}_{\left[1 c_{2} c_{3} \ldots c_{m}\right]}^{1}\right|=\left|\overline{\mathrm{O}}_{\left[\left(c_{2}+1\right) c_{3} \ldots c_{m}\right]}^{1}\right| .
$$

Lemma 1. For any given $s \in \mathbb{N}$, the ratio of the lengths of the cylindrical sets $\overline{\mathrm{O}}_{\left[c_{1} \ldots c_{m} s\right]}^{1}$ and $\overline{\mathrm{O}}_{\left[c_{1} \ldots c_{m}\right]}^{1}$ is

$$
\frac{\left|\overline{\mathrm{O}}_{\left[c_{1} \ldots c_{m} s\right]}^{1}\right|}{\left|\overline{\mathrm{O}}_{\left[c_{1} \ldots c_{m}\right]}^{1}\right|}=\frac{a}{(a+s-1)(a+s)}=f_{s}(a) \text {, }
$$

where $a=1+\sigma_{m}$. Moreover,

$$
f_{s}(a) \leq \frac{1}{2(2 s-1)}
$$


and for $m \geq s-1$,

$$
\frac{\left|\overline{\mathrm{O}}_{\left[c_{1} \ldots c_{m} s\right]}^{1}\right|}{\left|\overline{\mathrm{O}}_{\left[c_{1} \ldots c_{m}\right]}^{1}\right|} \leq \frac{m+1}{(m+s)(m+s+1)} .
$$

Proof. Equality (7) follows directly from (6). Consider

$$
f_{s}(x)=\frac{x}{(x+s-1)(x+s)}
$$

as a function of $x \geq 1$. This function increases on $[1, \sqrt{(s-1) s}]$ and decreases on $[\sqrt{(s-1) s}, \infty)$. Since $a$ takes only positive integer values, we have

$$
\max _{a \in \mathbb{N}} f_{s}(a)=f_{s}(s-1)=f_{s}(s)=\frac{1}{2(2 s-1)} .
$$

So, inequality (8) holds.

As $f_{s}(x)$ decreases on $(s, \infty)$, we have $f_{s}(a) \leq f_{s}(m+1)$, so inequality (9) holds.

COROllary. If $c_{1}+\cdots+c_{m}=s_{1}+\cdots+s_{k}$ then

$$
\frac{\left|\overline{\mathrm{O}}_{\left[c_{1} \ldots c_{m} s\right]}^{1}\right|}{\left|\overline{\mathrm{O}}_{\left[c_{1} \ldots c_{m}\right]}^{1}\right|}=\frac{\left|\overline{\mathrm{O}}_{\left[s_{1} \ldots s_{k} s\right]}^{1}\right|}{\left|\overline{\mathrm{O}}_{\left[s_{1} \ldots s_{k}\right]}^{1}\right|} \text {. }
$$

REMARK. Let $\Delta_{c_{1} \ldots c_{m}}^{\text {c.f. }}$ be a cylindrical set generated by the continued fraction representation of real numbers. It is well known (see, e.g., [6]) that

$$
\frac{\left|\Delta_{c_{1} . \ldots c_{m} s}^{\text {c.f. }}\right|}{\left|\Delta_{c_{1} \ldots c_{m}}^{\text {c.f. }}\right|}=\frac{1}{s^{2}} \cdot \frac{1+\frac{Q_{m-1}}{Q_{m}}}{\left(1+\frac{Q_{m-1}}{s Q_{m}}\right)\left(1+\frac{1}{s}+\frac{Q_{m-1}}{s Q_{m}}\right)},
$$

where $Q_{k}$ is the denominator of the $k$ th convergent of the continued fraction $\left[c_{1}, c_{2}, \ldots\right]$, i.e.,

$$
Q_{k}=c_{k} Q_{k-1}+Q_{k-2} \quad \text { with } \quad Q_{0}=1, Q_{1}=c_{1} .
$$

From (10) it follows that

$$
\frac{1}{3 s^{2}}<\frac{\left|\Delta_{c_{1} \ldots . . c_{m} s}^{\text {c.f. }}\right|}{\left|\Delta_{c_{1} \ldots c_{m}}^{\text {c.f. }}\right|}<\frac{2}{s^{2}}
$$

for any sequence $\left(c_{1}, \ldots, c_{m}\right)$ and for any $s \in \mathbb{N}$. For the $\overline{\mathrm{O}}^{1}$-representation we have $f_{s}(a) \rightarrow 0$ as $a \rightarrow \infty$, and Lemma 1 shows the fundamental difference between metric relations in the representation of numbers by the first Ostrogradsky series and by continued fractions.

Lemma 2. Let $\overline{\mathrm{O}}_{\left[c_{1} \ldots c_{m}\right]}^{1}$ be a fixed cylindrical set. Then

$$
\lambda\left(\bigcup_{s=1}^{k} \overline{\mathrm{O}}_{\left[c_{1} \ldots c_{m} s\right]}^{1}\right)=\frac{k}{\sigma_{m}+k+1}\left|\overline{\mathrm{O}}_{\left[c_{1} \ldots c_{m}\right]}^{1}\right| .
$$


Proof. From (6) it follows that

$$
\begin{aligned}
\lambda\left(\bigcup_{s=1}^{k} \overline{\mathrm{O}}_{\left[c_{1} \ldots c_{m} s\right]}^{1}\right) & =\sum_{s=1}^{k}\left|\overline{\mathrm{O}}_{\left[c_{1} \ldots c_{m} s\right]}^{1}\right| \\
& =\frac{1}{\sigma_{1} \ldots \sigma_{m}} \sum_{s=1}^{k} \frac{1}{\left(\sigma_{m}+s\right)\left(\sigma_{m}+s+1\right)} \\
& =\frac{1}{\sigma_{1} \ldots \sigma_{m}}\left(\frac{1}{\sigma_{m}+1}-\frac{1}{\sigma_{m}+k+1}\right) \\
& =\frac{1}{\sigma_{1} \ldots \sigma_{m}\left(\sigma_{m}+1\right)} \cdot \frac{k}{\sigma_{m}+k+1} \\
& =\left|\overline{\mathrm{O}}_{\left[c_{1} \ldots c_{m}\right]}^{1}\right| \frac{k}{\sigma_{m}+k+1},
\end{aligned}
$$

which proves Lemma 2.

Corollary 1. For any $k \in \mathbb{N}$ and for any sequence $\left(c_{1}, \ldots, c_{m}\right)$,

$$
\frac{1}{\sigma_{m}+2}\left|\overline{\mathrm{O}}_{\left[c_{1} \ldots c_{m}\right]}^{1}\right| \leq \lambda\left(\bigcup_{s=1}^{k} \overline{\mathrm{O}}_{\left[c_{1} \ldots c_{m} s\right]}^{1}\right) \leq \frac{k}{m+k+1}\left|\overline{\mathrm{O}}_{\left[c_{1} \ldots c_{m}\right]}^{1}\right| .
$$

REMARK. If $V \subset \mathbb{N}$, then it is evident that

$$
\sum_{s \in V}\left|\overline{\mathrm{O}}_{\left[c_{1} \ldots c_{m} s\right]}^{1}\right|=\left|\overline{\mathrm{O}}_{\left[c_{1} \ldots c_{m}\right]}^{1}\right|-\sum_{s \in \mathbb{N} \backslash V}\left|\overline{\mathrm{O}}_{\left[c_{1} \ldots c_{m} s\right]}^{1}\right| .
$$

COROLlary 2. Let $\overline{\mathrm{O}}_{\left[c_{1} \ldots c_{m}\right]}^{1}$ be a cylindrical set. Then

$$
\lambda\left(\bigcup_{c=k+1}^{\infty} \overline{\mathrm{O}}_{\left[c_{1} \ldots c_{m} c\right]}^{1}\right)=\frac{\sigma_{m}+1}{\sigma_{m}+k+1}\left|\overline{\mathrm{O}}_{\left[c_{1} \ldots c_{m}\right]}^{1}\right| .
$$

Corollary 3. For any $k \in \mathbb{N}$ and for any sequence $\left(c_{1}, \ldots, c_{m}\right)$

$$
\left.\frac{m+1}{m+k+1}\left|\overline{\mathrm{O}}_{\left[c_{1} \ldots c_{m}\right]}^{1}\right| \leq \lambda\left(\bigcup_{c=k+1}^{\infty} \overline{\mathrm{O}}_{\left[c_{1} \ldots c_{m} c\right]}^{1}\right) \leq \frac{\sigma_{m}+1}{\sigma_{m}+2} \mid \overline{\mathrm{O}}_{\left[c_{1} \ldots c_{m}\right]}^{1}\right] .
$$

2. The set $C\left[\overline{\mathrm{O}}^{1},\left\{V_{n}\right\}\right]$. In this section we shall study the metric properties of the set $C\left[\overline{\mathrm{O}}^{1},\left\{V_{n}\right\}\right]$, which is the closure of the set $\left\{x: g_{n}(x) \in\right.$ $\left.V_{n}, n \in \mathbb{N}\right\}$, consisting of the real numbers $x \in[0,1]$ whose $\overline{\mathrm{O}}^{1}$-symbols satisfy the condition $g_{n}(x) \in V_{n}$, where $\left\{V_{n}\right\}$ is a fixed sequence of nonempty subsets of $\mathbb{N}$.

It is evident that

(1) if $V_{n}=\mathbb{N}$ for all $n \in \mathbb{N}$, then $C\left[\overline{\mathrm{O}}^{1},\left\{V_{n}\right\}\right]=[0,1]$,

(2) if $V_{n}=\mathbb{N}$ for all $n>n_{0}$, then $C\left[\overline{\mathrm{O}}^{1},\left\{V_{n}\right\}\right]$ is a union of segments. 
We are interested only in the case where $V_{n} \neq \mathbb{N}$ for infinitely many $n$. Let

$$
F_{k}=\left(\bigcup_{c_{1} \in V_{1}} \ldots \bigcup_{c_{k} \in V_{k}} \overline{\mathrm{O}}_{\left[c_{1} \ldots c_{k}\right]}^{1}\right)^{\mathrm{cl}}
$$

where cl stands for closure, let $F_{0}=[0,1]$ and let $\bar{F}_{k+1}=F_{k} \backslash F_{k+1}$. It is not hard to prove that

$$
C\left[\overline{\mathrm{O}}^{1},\left\{V_{n}\right\}\right]=\bigcap_{k=1}^{\infty} F_{k} .
$$

It is a perfect set (that is, a closed set without isolated points). If $V_{n} \neq \mathbb{N}$ for infinitely many $n$, then it is a nowhere dense set. Then

$$
\begin{aligned}
\lambda\left(F_{k}\right) & =\sum_{c_{1} \in V_{1}} \ldots \sum_{c_{k} \in V_{k}} \frac{1}{\sigma_{1} \ldots \sigma_{k}\left(\sigma_{k}+1\right)}, \\
\lambda\left(\bar{F}_{k+1}\right) & \left.=\sum_{c_{1} \in V_{1}} \ldots \sum_{c_{k} \in V_{k}} \sum_{s \notin V_{k+1}} \frac{1}{\sigma_{1} \ldots \sigma_{k}\left(\sigma_{k}+s\right)\left(\sigma_{k}+s+1\right)}\right), \\
& =\sum_{c_{1} \in V_{1}} \ldots \sum_{c_{k} \in V_{k}}\left(\frac{1}{\sigma_{1} \ldots \sigma_{k}} \sum_{s \notin V_{k+1}} \frac{1}{\left(\sigma_{k}+s\right)\left(\sigma_{k}+s+1\right)}\right)
\end{aligned}
$$

and from the continuity of Lebesgue measure it follows that

$$
\lambda\left(C\left[\overline{\mathrm{O}}^{1},\left\{V_{n}\right\}\right]\right)=\lim _{k \rightarrow \infty} \lambda\left(F_{k}\right) .
$$

Lemma 3. The Lebesgue measure of $C\left[\overline{\mathrm{O}}^{1},\left\{V_{n}\right\}\right]$ is 0 if and only if

$$
\sum_{k=1}^{\infty} \frac{\lambda\left(\bar{F}_{k+1}\right)}{\lambda\left(F_{k}\right)}=\infty
$$

Proof. We have

$$
\begin{aligned}
\lambda\left(C\left[\overline{\mathrm{O}}^{1},\left\{V_{n}\right\}\right]\right) & =\lim _{k \rightarrow \infty} \lambda\left(F_{k+1}\right)=\lim _{k \rightarrow \infty} \frac{\lambda\left(F_{k+1}\right)}{\lambda\left(F_{k}\right)} \cdot \frac{\lambda\left(F_{k}\right)}{\lambda\left(F_{k-1}\right)} \cdot \ldots \cdot \frac{\lambda\left(F_{1}\right)}{\lambda\left(F_{0}\right)} \\
& =\prod_{k=0}^{\infty} \frac{\lambda\left(F_{k+1}\right)}{\lambda\left(F_{k}\right)}=\prod_{k=0}^{\infty} \frac{\lambda\left(F_{k}\right)-\lambda\left(\bar{F}_{k+1}\right)}{\lambda\left(F_{k}\right)} \\
& =\prod_{k=0}^{\infty}\left(1-\frac{\lambda\left(\bar{F}_{k+1}\right)}{\lambda\left(F_{k}\right)}\right)=0
\end{aligned}
$$

if and only if

$$
\sum_{k=1}^{\infty} \frac{\lambda\left(\bar{F}_{k+1}\right)}{\lambda\left(F_{k}\right)}=\infty
$$

since $0 \leq \lambda\left(\bar{F}_{k+1}\right) / \lambda\left(F_{k}\right)<1$. 
First of all we shall study the problem of determining the Lebesgue measure of $C\left[\overline{\mathrm{O}}^{1}, V\right]=C\left[\overline{\mathrm{O}}^{1},\left\{V_{n}\right\}\right]$ with $V_{n}=V$, a fixed proper subset of positive integers. The sets $C\left[\overline{\mathrm{O}}^{1}, V\right]$ with

(1) $V=\{1, \ldots, m\}$,

(2) $V=\{m+1, m+2, \ldots\}$,

(3) $V=\{1,3,5, \ldots\}$

are the simplest among $C\left[\overline{\mathrm{O}}^{1}, V\right]$.

Let us solve the first problem in a more general setting.

THEOREM 1. If $V_{k}$ contains $n_{k}$ symbols $(k \in \mathbb{N})$ and

$$
\varliminf_{k \rightarrow \infty} \frac{n_{1} \ldots n_{k}}{(k+1) !}=0
$$

then $\lambda\left(C\left[\overline{\mathrm{O}}^{1},\left\{V_{k}\right\}\right]\right)=0$.

Proof. From the properties of cylindrical sets it follows that

$$
\lambda\left(F_{k}\right)=\sum_{\substack{v_{i} \in V_{i} \\ i=1, k}}\left|\overline{\mathrm{O}}_{\left[v_{1} \ldots v_{k}\right]}^{1}\right| \leq \frac{n_{1} \ldots n_{k}}{(k+1) !}
$$

and

$$
\lambda\left(C\left[\overline{\mathrm{O}}^{1},\left\{V_{k}\right\}\right]\right)=\lim _{k \rightarrow \infty} \lambda\left(F_{k}\right) \leq \lim _{k \rightarrow \infty} \frac{n_{1} \ldots n_{k}}{(k+1) !}=0 .
$$

Corollary. If $n_{k} \leq m$ (for any $k \in \mathbb{N}$ ) for some fixed $m$, then we have $\lambda\left(C\left[\overline{\mathrm{O}}^{1},\left\{V_{k}\right\}\right]\right)=0$.

Theorem 2. Let $V_{k}=\left\{1, \ldots, m_{k}\right\}, m_{k} \in \mathbb{N}$. If $\sum_{k=1}^{\infty} 1 / m_{k}=\infty$, then $\lambda\left(C\left[\overline{\mathrm{O}}^{1},\left\{V_{n}\right\}\right]\right)=0$.

Proof. Let $\overline{\mathrm{O}}_{\left[c_{1} \ldots c_{k}\right]}^{1}$ be a fixed cylindrical set of rank $k$. Then

$$
\begin{aligned}
\sum_{c \notin V_{k+1}}\left|\overline{\mathrm{O}}_{\left(c_{1} \ldots c_{k} c\right)}^{1}\right| & =\frac{1}{\sigma_{1} \ldots \sigma_{k}} \sum_{c=m_{k+1}+1}^{\infty} \frac{1}{\left(\sigma_{k}+c\right)\left(\sigma_{k}+c+1\right)} \\
& =\frac{1}{\sigma_{1} \ldots \sigma_{k}\left(\sigma_{k}+m_{k+1}+1\right)} .
\end{aligned}
$$

Since

$$
\frac{1}{\sigma_{k}+m_{k+1}+1}>\frac{1}{\left(m_{k+1}+1\right)\left(\sigma_{k}+1\right)}
$$

we have

$$
\sum_{c \notin V_{k+1}}\left|\overline{\mathrm{O}}_{\left(c_{1} \ldots c_{k} c\right)}^{1}\right|>\frac{1}{m_{k+1}+1}\left|\overline{\mathrm{O}}_{\left[c_{1} \ldots c_{k}\right]}^{1}\right| .
$$


Summing over all $c_{1} \in V_{1}, \ldots, c_{k} \in V_{k}$, we have

$$
\lambda\left(\bar{F}_{k+1}\right)>\frac{1}{m_{k+1}+1} \lambda\left(F_{k}\right), \quad \text { i.e., } \quad \frac{\lambda\left(\bar{F}_{k+1}\right)}{\lambda\left(F_{k}\right)}>\frac{1}{m_{k+1}+1}
$$

for any $k \in \mathbb{N}$, and the statement follows directly from Lemma 3 .

Let $E$ be the set of all real numbers with bounded $\overline{\mathrm{O}}^{1}$-symbols, i.e., $x \in E$ iff there exists a constant $K_{x}$ such that $g_{k}(x) \leq K_{x}$ for all $k \in \mathbb{N}$.

Theorem 3. The Lebesgue measure of $E$ is 0 .

Proof. For $m \in \mathbb{N}$, consider the set $E_{m}=\left\{x: g_{k}(x) \leq m, \forall k \in \mathbb{N}\right\}$ of uniformly $m$-bounded symbols. It is not hard to see that $E_{m}=C\left[\overline{\mathrm{O}}^{1},\left\{V_{k}\right\}\right]$ with $V_{k}=\{1, \ldots, m\}$. From Theorem 2 it follows that $\lambda\left(C\left[\overline{\mathrm{O}}^{1},\left\{V_{k}\right\}\right]\right)=0$.

Since $E=\bigcup_{m=1}^{\infty} E_{m}$ and $\lambda\left(E_{m}\right)=0$, we have the desired conclusion.

Corollary. For Lebesgue almost all $x \in[0,1]$,

$$
\varlimsup_{k \rightarrow \infty} g_{k}(x)=\infty \text {. }
$$

Now consider the case where $V_{k}=\left\{v_{k}+1, v_{k}+2, \ldots\right\}$ and $\left\{v_{k}\right\}$ is a fixed sequence of positive integers.

LEMMA 4. Let $\overline{\mathrm{O}}_{\left[c_{1} \ldots c_{n}\right]}^{1}$ be a fixed cylindrical set or, if $n=0$, the unit interval $[0,1]$; let $\left\{v_{k}\right\}$ be a fixed sequence of positive integers, let $V_{k}=$ $\left\{v_{k}+1, v_{k}+2, \ldots\right\}$, and let

$$
\begin{aligned}
& F_{k}^{c_{1} \ldots c_{n}}:=F_{n+k} \cap \overline{\mathrm{O}}_{\left[c_{1} \ldots c_{n}\right]}^{1}=\bigcup_{c_{n+1}>v_{n+1}} \ldots \bigcup_{c_{n+k}>v_{n+k}} \overline{\mathrm{O}}_{\left[c_{1} \ldots c_{n} c_{n+1} \ldots c_{n+k}\right]}^{1}, \\
& \bar{F}_{k+1}^{c_{1} \ldots c_{n}}:=F_{k}^{c_{1} \ldots c_{n}} \backslash F_{k+1}^{c_{1} \ldots c_{n}}=\bigcup_{c_{n+1}>v_{n+1}} \ldots \bigcup_{c_{n+k}>v_{n+k}} \bigcup_{s=1}^{v_{n+k+1}} \overline{\mathrm{O}}_{\left(c_{1} \ldots c_{n} c_{n+1} \ldots c_{n+k} s\right)}^{1} .
\end{aligned}
$$

Then

$$
\frac{\lambda\left(\bar{F}_{k+1}^{c_{1} \ldots c_{n}}\right)}{\lambda\left(\bar{F}_{k}^{c_{1} \ldots c_{n}}\right)}<\frac{1}{2} \cdot \frac{v_{n+k+1}}{v_{n+k}} .
$$

Proof. Let $\overline{\mathrm{O}}_{\left(c_{1} \ldots c_{n+k-1}\right)}^{1}$ be a cylindrical interval of rank $n+k-1$. Then

$$
\begin{aligned}
\sum_{s \notin V_{n+k}}\left|\overline{\mathrm{O}}_{\left(c_{1} \ldots c_{n+k-1} s\right)}^{1}\right| & =\sum_{s=1}^{v_{n+k}} \frac{1}{\sigma_{1} \ldots \sigma_{n+k-1}\left(\sigma_{n+k-1}+s\right)\left(\sigma_{n+k-1}+s+1\right)} \\
& =\frac{1}{\sigma_{1} \ldots \sigma_{n+k-1}}\left(\frac{1}{\sigma_{n+k-1}+1}-\frac{1}{\sigma_{n+k-1}+v_{n+k}+1}\right) \\
& =\frac{v_{n+k}}{\sigma_{1} \ldots \sigma_{n+k-1}\left(\sigma_{n+k-1}+1\right)\left(\sigma_{n+k-1}+v_{n+k}+1\right)} .
\end{aligned}
$$


For the same cylindrical interval we have

$$
\begin{aligned}
& \sum_{l \in V_{n+k}} \sum_{s \notin V_{n+k+1}}\left|\overline{\mathrm{O}}_{\left(c_{1} \ldots c_{n+k-1} l s\right)}^{1}\right| \\
&=\sum_{l=v_{n+k}+1}^{\infty} \frac{1}{\sigma_{1} \ldots \sigma_{n+k-1}\left(\sigma_{n+k-1}+l\right)}\left(\frac{1}{\sigma_{n+k-1}+l+1}\right. \\
&\left.-\frac{1}{\sigma_{n+k-1}+l+v_{n+k+1}+1}\right) \\
&= \frac{1}{\sigma_{1} \ldots \sigma_{n+k-1}} \sum_{l=v_{n+k}+1}^{\infty}\left(\frac{1}{\left(\sigma_{n+k-1}+l\right)\left(\sigma_{n+k-1}+l+1\right)}\right. \\
&= \frac{1}{\sigma_{1} \ldots \sigma_{n+k-1}}\left(\frac{1}{\left.\sigma_{n+k-1}+l\right)\left(\sigma_{n+k-1}+l+v_{n+k+1}+1\right)}\right) \\
&= \frac{1}{\sigma_{n+k-1}+v_{n+k}+1} \\
& v_{n+k+1}+1 \frac{1}{\sigma_{1} \ldots \sigma_{n+k-1}\left(\sigma_{n+k-1}+1\right)\left(\sigma_{n+k-1}+v_{n+k}+1\right)} X_{k} .
\end{aligned}
$$

Let us estimate the expression

$$
\begin{aligned}
X_{k}= & \frac{\left(\sigma_{n+k-1}+1\right)\left(\sigma_{n+k-1}+v_{n+k}+1\right)}{v_{n+k}}\left(\frac{1}{\sigma_{n+k-1}+v_{n+k}+1}\right. \\
& \left.-\frac{1}{1+v_{n+k+1}} \sum_{i=1}^{1+v_{n+k+1}} \frac{1}{\sigma_{n+k-1}+v_{n+k}+i}\right) \\
= & \frac{\sigma_{n+k-1}+1}{v_{n+k}}\left(1-\frac{1}{1+v_{n+k+1}} \sum_{i=1}^{1+v_{n+k+1}} \frac{\sigma_{n+k-1}+v_{n+k}+1}{\sigma_{n+k-1}+v_{n+k}+i}\right) \\
= & \frac{\sigma_{n+k-1}+1}{v_{n+k}}\left(1-\frac{1}{1+v_{n+k+1}} \sum_{i=1}^{1+v_{n+k+1}}\left(1-\frac{i-1}{\sigma_{n+k-1}+v_{n+k}+i}\right)\right) \\
= & \frac{\sigma_{n+k-1}+1}{v_{n+k}} \cdot \frac{1}{1+v_{n+k+1}} \sum_{i=2}^{1+v_{n+k+1}} \frac{i-1}{\sigma_{n+k-1}+v_{n+k}+i} .
\end{aligned}
$$

Now let us estimate the sum

$$
\frac{1}{n_{0}+1}+\frac{2}{n_{0}+2}+\cdots+\frac{m_{k}}{n_{0}+m_{k}},
$$


where $n_{0}$ and $m_{k}>1$ are positive integers. Let

$$
C_{k}:=\frac{1}{n_{0}+1}+\frac{1}{n_{0}+2}+\cdots+\frac{1}{n_{0}+m_{k}}
$$

and consider the matrix

$$
\left[\begin{array}{ccccc}
\frac{1}{n_{0}+1} & \frac{1}{n_{0}+2} & \frac{1}{n_{0}+3} & \cdots & \frac{1}{n_{0}+m_{k}} \\
\frac{1}{n_{0}+1} & \frac{1}{n_{0}+2} & \frac{1}{n_{0}+3} & \cdots & \frac{1}{n_{0}+m_{k}} \\
\frac{1}{n_{0}+1} & \frac{1}{n_{0}+2} & \frac{1}{n_{0}+3} & \cdots & \frac{1}{n_{0}+m_{k}} \\
\vdots & \vdots & \vdots & \vdots & \vdots \\
\frac{1}{n_{0}+1} & \frac{1}{n_{0}+2} & \frac{1}{n_{0}+3} & \cdots & \frac{1}{n_{0}+m_{k}}
\end{array}\right] .
$$

The sum of its elements is $m_{k} C_{k}$. The sum of all elements on the main diagonal is $C_{k}$. The sum of all elements above the diagonal is less than the sum of those below the diagonal (for any element above the diagonal, the symmetrical element is greater).

The sum of all off-diagonal elements is $\left(m_{k}-1\right) C_{k}$. So, the sum of all elements above the diagonal is less than $\left(m_{k}-1\right) C_{k} / 2$, and the sum of all elements above or on the diagonal is equal to

$$
\frac{1}{n_{0}+1}+\frac{2}{n_{0}+2}+\cdots+\frac{m_{k}}{n_{0}+m_{k}}<\frac{m_{k}-1}{2} C_{k}+C_{k}=\frac{m_{k}+1}{2} C_{k} .
$$

So,

$$
\begin{aligned}
\frac{1}{n_{0}+1}+\frac{2}{n_{0}+2}+\cdots & +\frac{m_{k}}{n_{0}+m_{k}} \\
& <\frac{m_{k}+1}{2}\left(\frac{1}{n_{0}+1}+\frac{1}{n_{0}+2}+\cdots+\frac{1}{n_{0}+m_{k}}\right) .
\end{aligned}
$$

Therefore,

$$
\begin{aligned}
X_{k} & =\frac{\sigma_{n+k-1}+1}{v_{n+k}} \cdot \frac{1}{1+v_{n+k+1}} \sum_{i=1}^{v_{n+k+1}} \frac{i}{\left(\sigma_{n+k-1}+v_{n+k}+1\right)+i} \\
& <\frac{\sigma_{n+k-1}+1}{v_{n+k}} \cdot \frac{1}{1+v_{n+k+1}} \cdot \frac{v_{n+k+1}+1}{2} \sum_{i=1}^{v_{n+k+1}} \frac{1}{\sigma_{n+k-1}+v_{n+k}+i+1} \\
& =\frac{1}{2 v_{n+k}} \sum_{i=1}^{v_{n+k+1}} \frac{\sigma_{n+k-1}+1}{\sigma_{n+k-1}+v_{n+k}+i+1}<\frac{1}{2} \cdot \frac{v_{n+k+1}}{v_{n+k}} .
\end{aligned}
$$

So,

$$
\sum_{l \in V_{n+k}} \sum_{s \notin V_{n+k+1}}\left|\overline{\mathrm{O}}_{\left(c_{1} \ldots c_{n+k-1} l s\right)}^{1}\right|<\frac{1}{2} \cdot \frac{v_{n+k+1}}{v_{n+k}} \sum_{l \notin V_{n+k}}\left|\overline{\mathrm{O}}_{\left(c_{1} \ldots c_{n+k-1} l\right)}^{1}\right| .
$$


Summing over all $c_{n+1} \in V_{n+1}, \ldots, c_{n+k-1} \in V_{n+k-1}$, we have

$$
\lambda\left(\bar{F}_{k+1}^{c_{1} \ldots c_{n}}\right)<\frac{1}{2} \cdot \frac{v_{n+k+1}}{v_{n+k}} \lambda\left(\bar{F}_{k}^{c_{1} \ldots c_{n}}\right)
$$

which proves the lemma.

Corollary 1. Let $V_{k}=\left\{v_{k}+1, v_{k}+2, \ldots\right\}, v_{k} \in \mathbb{N}$. Then

$$
\lambda\left(\bar{F}_{k+1}\right)<\frac{1}{2} \cdot \frac{v_{k+1}}{v_{k}} \lambda\left(\bar{F}_{k}\right) .
$$

Corollary 2. Let $V_{k}=V=\{m+1, m+2, \ldots\}, m \in \mathbb{N}$. Then

$$
\lambda\left(\bar{F}_{k+1}^{c_{1} \ldots c_{n}}\right)<\frac{1}{2} \lambda\left(\bar{F}_{k}^{c_{1} \ldots c_{n}}\right)
$$

for any positive integer $k$ and any $c_{1} \in V, \ldots, c_{n} \in V$, and therefore,

$$
\lambda\left(\bar{F}_{k+1}\right)<\frac{1}{2} \lambda\left(\bar{F}_{k}\right) .
$$

ThEOREM 4. Let $\left\{v_{k}\right\}$ be a fixed sequence of positive integers, and let

$$
V_{k}=\left\{v_{k}+1, v_{k}+2, \ldots\right\} .
$$

If there exists $k_{0} \in \mathbb{N}$ such that

$$
v_{k+1} / v_{k} \leq C_{0}<2 \text { for any } k>k_{0},
$$

then $\lambda\left(C\left[\overline{\mathrm{O}}^{1},\left\{V_{k}\right\}\right]\right)>0$.

Proof. Fix $\overline{\mathrm{O}}_{\left[c_{1} \ldots c_{n}\right]}^{1}$ with $n>k_{0}$ and $c_{i} \in V_{i}$. We shall prove that the set

$$
\Delta_{c_{1} \ldots c_{n}}=C\left[\overline{\mathrm{O}}^{1},\left\{V_{k}\right\}\right] \cap \overline{\mathrm{O}}_{\left[c_{1} \ldots c_{n}\right]}^{1}
$$

has positive Lebesgue measure. To this end, consider $\overline{\mathrm{O}}_{\left[c_{1} \ldots c_{n+1}\right]}^{1}, c_{n+1}>$ $v_{n+1}$, and the corresponding subset

$$
\Delta_{c_{1} \ldots c_{n+1}}=C\left[\overline{\mathrm{O}}^{1},\left\{V_{k}\right\}\right] \cap \overline{\mathrm{O}}_{\left[c_{1} \ldots c_{n+1}\right]}^{1} .
$$

From Lemma 4 it follows that

$$
\begin{aligned}
& \lambda\left(\bar{F}_{k+1}^{c_{1} \ldots c_{n+1}}\right)<\frac{1}{2} \cdot \frac{v_{n+k+1}}{v_{n+k}} \lambda\left(\bar{F}_{k}^{c_{1} \ldots c_{n+1}}\right) \leq \frac{1}{2} C_{0} \lambda\left(\bar{F}_{k}^{c_{1} \ldots c_{n+1}}\right) \\
& \quad<\frac{1}{2} C_{0} \cdot \frac{1}{2} \cdot \frac{v_{n+k}}{v_{n+k-1}} \lambda\left(\bar{F}_{k-1}^{c_{1} \ldots c_{n+1}}\right) \leq\left(\frac{C_{0}}{2}\right)^{2} \lambda\left(\bar{F}_{k-1}^{c_{1} \ldots c_{n+1}}\right) \cdots \\
& \quad \leq\left(C_{0} / 2\right)^{k} \lambda\left(\bar{F}_{1}^{c_{1} \ldots c_{n+1}}\right)
\end{aligned}
$$

for any $k \in \mathbb{N}$. Using Lemma 2 , we have

$$
\lambda\left(\bar{F}_{1}^{c_{1} \ldots c_{n+1}}\right)=\sum_{s=1}^{v_{n+2}}\left|\overline{\mathrm{O}}_{\left(c_{1} \ldots c_{n+1} s\right)}^{1}\right|=\frac{v_{n+2}}{\sigma_{n+1}+v_{n+2}+1} \cdot\left|\overline{\mathrm{O}}_{\left[c_{1} \ldots c_{n+1}\right]}^{1}\right| .
$$


So,

$$
\begin{aligned}
\lambda\left(\Delta_{c_{1} \ldots c_{n+1}}\right) & =\left|\overline{\mathrm{O}}_{\left[c_{1} \ldots c_{n+1}\right]}^{1}\right|-\sum_{k=1}^{\infty} \lambda\left(\bar{F}_{k}^{c_{1} \ldots c_{n+1}}\right) \\
& >\left|\overline{\mathrm{O}}_{\left[c_{1} \ldots c_{n+1}\right]}^{1}\right|-\sum_{k=1}^{\infty}\left(C_{0} / 2\right)^{k-1} \lambda\left(\bar{F}_{1}^{c_{1} \ldots c_{n+1}}\right) \\
& =\left|\overline{\mathrm{O}}_{\left[c_{1} \ldots c_{n+1}\right]}^{1}\right| \cdot\left(1-\frac{2}{2-C_{0}} \cdot \frac{v_{n+2}}{\sigma_{n+1}+v_{n+2}+1}\right) .
\end{aligned}
$$

Since the numbers $c_{1}, \ldots, c_{n}, v_{n+2}, C_{0}$ are fixed, and $c_{n+1}>v_{n+1}$, there exists $c^{*} \in \mathbb{N}$ such that

$$
1-\frac{2}{2-C_{0}} \cdot \frac{v_{n+2}}{\sigma_{n+1}+v_{n+2}+1}>0
$$

for any $c_{n+1}>c^{*}$. Hence, $\lambda\left(\Delta_{c_{1} \ldots c_{n+1}}\right)>0$ for any $c_{n+1}>c^{*}$, and therefore,

$$
\lambda\left(C\left[\overline{\mathrm{O}}^{1},\left\{V_{k}\right\}\right]\right)>\lambda\left(\Delta_{c_{1} \ldots c_{n}}\right)>\lambda\left(\Delta_{c_{1} \ldots c_{n+1}}\right)>0 .
$$

Corollary 1. Let $P_{n}(x)=a_{n} x^{n}+a_{n-1} x^{n-1}+\cdots+a_{1} x^{1}+a_{0}$ with $n \in \mathbb{N}$, $a_{i} \in \mathbb{Z}$ and $P_{n}(x)>0$ for any $x \in \mathbb{N}$. If $v_{k}=P_{n}(k)$, then $\lambda\left(C\left[\overline{\mathrm{O}}^{1},\left\{V_{k}\right\}\right]\right)>0$.

Corollary 2. If the sequence $\left\{v_{k}\right\}$ is bounded, then $\lambda\left(C\left[\overline{\mathrm{O}}^{1},\left\{V_{k}\right\}\right]\right)$ $>0$.

REMARK. Let us compare Theorem 4 with the corresponding proposition from the theory of continued fractions. Let $C\left[\right.$ c.f., $\left.\left\{V_{n}\right\}\right]$ be the closure of the set of all real numbers

$$
x=\left[a_{1}(x), a_{2}(x), \ldots\right],
$$

whose continued fraction's elements $a_{n}(x)$ satisfy $a_{n}(x) \in V_{n}$ for any $n \in \mathbb{N}$ (here $\left\{V_{n}\right\}$ is a fixed sequence of nonempty subsets of $\mathbb{N}$ as above). For example, if $V_{n}=V=\mathbb{N} \backslash\{1\}$ for any $n \in \mathbb{N}$, then $\lambda\left(C\left[\right.\right.$ c.f., $\left.\left.\left\{V_{n}\right\}\right]\right)=0$ (see, e.g., $[6,12])$, but $\lambda\left(C\left[\overline{\mathrm{O}}^{1},\left\{V_{n}\right\}\right]\right)>0$. So, Theorem 4 indicates an essential difference between the metric theories of continued fractions and $\overline{\mathrm{O}}^{1}$-representations.

Theorem 5. Let $m \in \mathbb{N}$ and $V=\mathbb{N} \backslash\{1, \ldots, m\}$. Then

$$
\lambda\left(C\left[\overline{\mathrm{O}}^{1}, V\right]\right)>\frac{1}{(m+1)^{2}} .
$$

Proof. Consider an arbitrary cylindrical set $\overline{\mathrm{O}}_{\left[c_{1}\right]}^{1}$ such that $c_{1} \in V$. From Corollary 2 to Lemma 4 it follows that

$$
\lambda\left(\bar{F}_{k+1}^{c_{1}}\right)<\frac{1}{2^{k}} \lambda\left(\bar{F}_{1}^{c_{1}}\right) .
$$


So, we have

$$
\lambda\left(\Delta_{c_{1}}\right)=\left|\overline{\mathrm{O}}_{\left[c_{1}\right]}^{1}\right|-\sum_{k=1}^{\infty} \lambda\left(\bar{F}_{k}^{c_{1}}\right)>\left|\overline{\mathrm{O}}_{\left[c_{1}\right]}^{1}\right|-\lambda\left(\bar{F}_{1}^{c_{1}}\right) \sum_{k=0}^{\infty} \frac{1}{2^{k}}=\left|\overline{\mathrm{O}}_{\left[c_{1}\right]}^{1}\right|-2 \lambda\left(\bar{F}_{1}^{c_{1}}\right) .
$$

Since

$$
\left.\lambda\left(\bar{F}_{1}^{c_{1}}\right)=\sum_{c=1}^{m}\left|\overline{\mathrm{O}}_{\left(c_{1} c\right)}^{1}\right|=\frac{m}{c_{1}+m+1}\left|\overline{\mathrm{O}}_{\left[c_{1}\right]}^{1}\right| \leq \frac{m}{2 m+2} \mid \overline{\mathrm{O}}_{\left[c_{1}\right]}^{1}\right]
$$

it follows that

$$
\lambda\left(\Delta_{c_{1}}\right)>\frac{1}{m+1}\left|\overline{\mathrm{O}}_{\left[c_{1}\right]}^{1}\right|
$$

So,

$$
\lambda\left(C\left[\overline{\mathrm{O}}^{1}, V\right]\right)=\sum_{c_{1}=m+1}^{\infty} \lambda\left(\Delta_{c_{1}}\right)>\frac{1}{m+1} \sum_{c_{1}=m+1}^{\infty}\left|\overline{\mathrm{O}}_{\left[c_{1}\right]}^{1}\right|=\frac{1}{(m+1)^{2}} .
$$

Finally, consider a more general case where $V_{k}=V=\mathbb{N} \backslash\left\{a_{1}, a_{2}, \ldots\right\}$ and $\left\{a_{n}\right\}$ is an arbitrary increasing sequence of positive integers.

THEOREM 6. Let $\left\{a_{n}\right\}$ be an increasing sequence of positive integers with $a_{n+1}-a_{n} \leq d$ for some fixed positive integer $d \geq 2$, and any $n \in \mathbb{N}$. If $V_{k}=V=\mathbb{N} \backslash\left\{a_{1}, a_{2}, \ldots\right\}$, then $\lambda\left(C\left[\overline{\mathrm{O}}^{1}, V\right]\right)=0$.

Proof. Fix a cylindrical set $\overline{\mathrm{O}}_{\left[c_{1} \ldots c_{k}\right]}^{1}$. Then

$$
\begin{aligned}
\sum_{c \notin V}\left|\overline{\mathrm{O}}_{\left(c_{1} \ldots c_{k} c\right)}^{1}\right| & =\frac{1}{\sigma_{1} \ldots \sigma_{k}} \sum_{n=1}^{\infty} \frac{1}{\left(\sigma_{k}+a_{n}\right)\left(\sigma_{k}+a_{n}+1\right)} \\
& >\frac{1}{\sigma_{1} \ldots \sigma_{k}} \sum_{n=1}^{\infty} \frac{1}{\left(\sigma_{k}+a_{n}^{\prime}\right)\left(\sigma_{k}+a_{n}^{\prime}+d\right)}=\frac{1}{d} \cdot \frac{1}{\sigma_{1} \ldots \sigma_{k}\left(\sigma_{k}+a_{1}\right)},
\end{aligned}
$$

where $a_{1}^{\prime}=a_{1}$ and $a_{n+1}^{\prime}=a_{n}^{\prime}+d \geq a_{n+1}$ for any positive integer $n$. Since

we have

$$
\frac{1}{\sigma_{k}+a_{1}} \geq \frac{1}{a_{1}\left(\sigma_{k}+1\right)}
$$

$$
\sum_{c \notin V}\left|\overline{\mathrm{O}}_{\left(c_{1} \ldots c_{k} c\right)}^{1}\right|>\frac{1}{a_{1} d}\left|\overline{\mathrm{O}}_{\left[c_{1} \ldots c_{k}\right]}^{1}\right| \text {. }
$$

Summing over all $c_{1} \in V, \ldots, c_{k} \in V$, we have

$$
\lambda\left(\bar{F}_{k+1}\right)>\frac{1}{a_{1} d} \lambda\left(F_{k}\right), \quad \text { i.e., } \quad \frac{\lambda\left(\bar{F}_{k+1}\right)}{\lambda\left(F_{k}\right)}>\frac{1}{a_{1} d}
$$

for any $k \in \mathbb{N}$, and the statement follows directly from Lemma 3 .

Corollary 1. If $V_{k}=V=\left\{b_{1}, b_{2}, \ldots\right\}$ with $b_{n+1}-b_{n} \geq 2$, then $\lambda\left(C\left[\overline{\mathrm{O}}^{1}, V\right]\right)=0$.

Corollary 2. If $V=\{1,3,5, \ldots\}$ or $V=\{2,4,6, \ldots\}$ then $\lambda\left(C\left[\overline{\mathrm{O}}^{1}, V\right]\right)$ $=0$. 
Acknowledgements. This work was supported by DFG 436 UKR 113/78, 113/80, SFB-611 projects and by the Alexander von Humboldt Foundation. The last three named authors gratefully acknowledge the hospitality of the Institute for Applied Mathematics of the University of Bonn.

\section{References}

[1] S. Albeverio, M. Pratsiovytyi, and G. Torbin, Singular probability distributions and fractal properties of sets of real numbers defined by the asymptotic frequencies of their s-adic digits, Ukrain. Math. J. 57 (2005), 1361-1370.

$[2]-,-,-$, Topological and fractal properties of real numbers which are not normal, Bull. Sci. Math. 129 (2005), 615-630.

[3] O. M. Baranovskyi, On some problems of metric theory of numbers defined by the first Ostrogradsky series, Trans. National Pedagog. Univ. of Ukraine Phys.-Math. Sci. 3 (2002), 391-402 (in Ukrainian).

[4] P. I. Bodnarchuk and V. Ya. Skorobogat'ko, Branching Continued Fractions and Their Applications, Naukova Dumka, Kyiv, 1974 (in Ukrainian).

[5] S. Kalpazidou, A. Knopfmacher, and J. Knopfmacher, Lüroth-type alternating series representations for real numbers, Acta Arith. 55 (1990), 311-322.

[6] A. Ya. Khintchine, Continued Fractions, Noordhoff, Groningen, 1963.

[7] Yu. V. Melnichuk, p-adic continued fractions, generated by algorithms of Euclid and Ostrogradsky, in: Computational Mathematics in Modern Scientific and Technical Progress (Kanev, 1974), 259-265 (in Russian).

[8] -, On representation of real numbers by fast converging series, in: Continued Fractions and Their Applications, Inst. Mat., Akad. Nauk Ukrain. SSR, Kiev, 1976, 77-78 (in Russian).

[9] L. Olsen, Applications of multifractal divergence points to sets of numbers defined by their N-adic expansion, Math. Proc. Cambridge Philos. Soc. 136 (2004), 139-165.

[10] -, Applications of multifractal divergence points to some sets of d-tuples of numbers defined by their N-adic expansion, Bull. Sci. Math. 128 (2004), 265-289.

[11] T. A. Pierce, On an algorithm and its use in approximating roots of algebraic equations, Amer. Math. Monthly 36 (1929), 523-525.

[12] M. V. Pratsiovytyi, Fractal approach to investigations of singular probability distributions, National Pedagogical Univ., Kyiv, 1998 (in Ukrainian).

[13] M. V. Pratsiovytyi and O. M. Baranovskyi, Applications of the Ostrogradsky series for the analytical description of distributions of random variables and mappings, in: Dynamical Systems: Proc. Ukrainian Mathematical Congress-2001, Inst. Math. NASU, Kyiv, 2003, 59-76 (in Ukrainian).

[14] - - - Properties of distributions of random variables with independent differences of consecutive elements of the Ostrogradskiu series, Teor. İmovīr. Mat. Stat. 2004, no. 70, 131-143 (in Ukrainian); English transl.: Theory Probab. Math. Statist. 2005, no. $70,147-160$.

[15] M. Pratsiovytyi and G. Torbin, Superfractality of the set of numbers having no frequency of n-adic digits, and fractal probability distributions, Ukrain. Math. J. 47 (1995), 1113-1118.

[16] E. Ya. Remez, On series with alternating signs which may be connected with two algorithms of $M$. V. Ostrogradski for approximation of irrational numbers, Uspekhi Mat. Nauk 6 (1951), no. 5, 33-42 (in Russian). 
[17] F. Schweiger, Ergodic Theory of Fibred Systems and Metric Number Theory, Oxford Sci. Publ., Oxford Univ. Press, New York, 1995.

[18] W. Sierpiński, Sur quelques algorithmes pour développer les nombres réels en séries, in: Oeuvres choisies, t. I, PWN, Warszawa, 1974, 236-254.

[19] K. G. Valēēv and E. D. Zlēbov, The metric theory of the Ostrogradskiu algorithm, Ukrain. Math. J. 27 (1975), 47-51.

Sergio Albeverio

Institut für Angewandte Mathematik

Universität Bonn

Wegelerstr. 6

D-53115 Bonn, Germany

E-mail: albeverio@uni-bonn.de

and

IZKS, Römerstr. 164, D-53117 Bonn, Germany

Mykola Pratsiovytyi

National Pedagogical University

Pyrogova St. 9

01030 Kyiv, Ukraine

and

Institute for Mathematics of NASU

Tereshchenkivs'ka St. 3

01601 Kyiv, Ukraine

E-mail: m_pratz@ukr.net
Oleksandr Baranovskyi Institute for Mathematics of NASU

Tereshchenkivs'ka St. 3

01601 Kyiv, Ukraine

E-mail: ombaranovskyi@ukr.net

Grygoriy Torbin

Institut für Angewandte Mathematik

Universität Bonn

Wegelerstr. 6

D-53115 Bonn, Germany

E-mail: torbin@wiener.iam.uni-bonn.de

and

National Pedagogical University

Pyrogova St. 9

01030 Kyiv, Ukraine

Received on 17.7.2006

and in revised form on 8.9.2007 